\title{
Speed measurements with a continuous wave Lidar prototype
}

\author{
Constantino Muñoz, Alejandro Rodríguez, Adolf Comerón, Òscar Batet, David Garcia, Francesc Rocadenbosch, \\ Michaël Sicard. \\ Universitat Politècnica de Catalunya (UPC), Dep. of Signal Theory and Communications (TSC), \\ Remote Sensing Lab (RSLAB), C/Jordi Girona, 1-3, D4-016, \\ 08034 Barcelona, SPAIN. \\ constan@tsc.upc.edu
}

\begin{abstract}
The UPC lidar group is developing a Doppler wind lidar based on the "edge-technique" [1]. Such systems use the slope of a high resolution optical filter as frequency discriminator. A laboratory prototype, able to measure the speed of solid targets emitting continuous wave radiation and using a Fabry-Perot interferometer as optical filter, has been built. A tuning control has been included to compensate drifts between the emitted frequency and the filter resonance frequencies in order to assure maximum sensitive measurements. The design of the system and different results concerning the performance of the tuning control system and speed measurements will be presented.
\end{abstract}

Keywords-Lidar; Doppler; Fabry-Perot; optic fiber

\section{INTRODUCTION}

The final objective of this work is to build a Doppler wind lidar system. Such systems measure the component of the wind velocity along the instrument line of sight with spatial resolution. They illuminate a region of the atmosphere using pulsed laser radiation and detect, in each resolution cell, the Doppler shift in the radiation backscattered either by molecules or by the aerosol particles drawn by the wind. Different techniques can be used to detect the Doppler frequency shift. Non-coherent or 'direct' detection techniques, based on optical interferometer frequency discrimination, are not as sensitive as coherent ones but, on the other hand, they are more suitable for systems operating in regions where atmospheric aerosol content is low [2] and they are also less demanding with optics [2] and laser characteristics. Chanin et al, in 1989 [3], proposed and used a first detection technique using optical filters as frequency discriminators. The 'edge technique', proposed by Korb et al in 1992 [1], is the one used in the UPC Doppler Lidar system.

In order to assess this measuring technique and to test the performance of all the subsystems in controlled conditions, avoiding those difficulties related with the use of pulsed light and with the detection of atmospheric signals, a continuouswave solid-target prototype has been designed and built. A prototype description, design calculations and several results are going to be presented.

\section{PRINCIPLE OF OPERATION}

As Korb et al presented [1], the edge technique principle consists in tuning a high-resolution optical filter so that the wavelength of the transmitting laser is located on the most sensitive zone of the slope of the filter, which produces big changes in transmission for small frequency shifts (in this case those produced by Doppler shift). The detection system has to measure the transmittance of the filter, alternatively, at two different frequencies: that of the emitted light and that of the received one, which is Doppler shifted, in every resolution cell. From these values and using a previous calibration of the filter frequency response, the Doppler shift and the speed of the target that has provoked it can be derived. This principle is shown in figure 1.

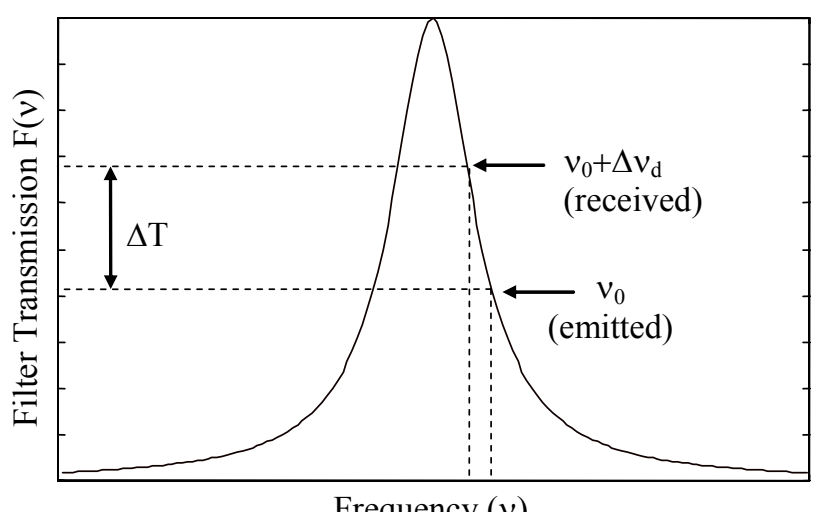

Figure 1. The edge technique principle.

\section{CONTINUOUS-WAVE SOLID-TARGET PROTOTYPE}

\section{A. Prototype Description}

Figure 2 shows the elements of the system used to illuminate the target and collect part of the reflected light and a sample of the emitted one. The transmitting continuous-wave laser is the seeder of a Spectra Physics GCR-190 laser, which will be used for the final wind measurements. After having been collimated and reduced, a beamsplitter (BS1) splits the 
output beam in two: one is sent towards the target and the other is used as the reference of the emitted frequency during the differential measurement (reference channel). A polarizing beam splitter (BS2), a quarter wave plate and a focusing system allow illuminating a spot on the paper surface of a rotating wheel, collecting part of the frequency shifted backscattered radiation and leading it to the measuring subsystem (Doppler channel).

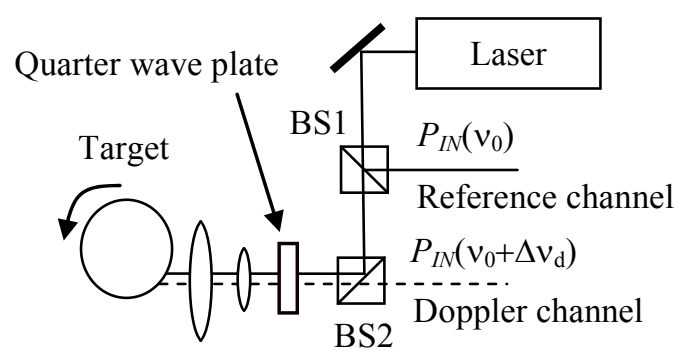

Figure 2. The emitting - receiving subsystem.

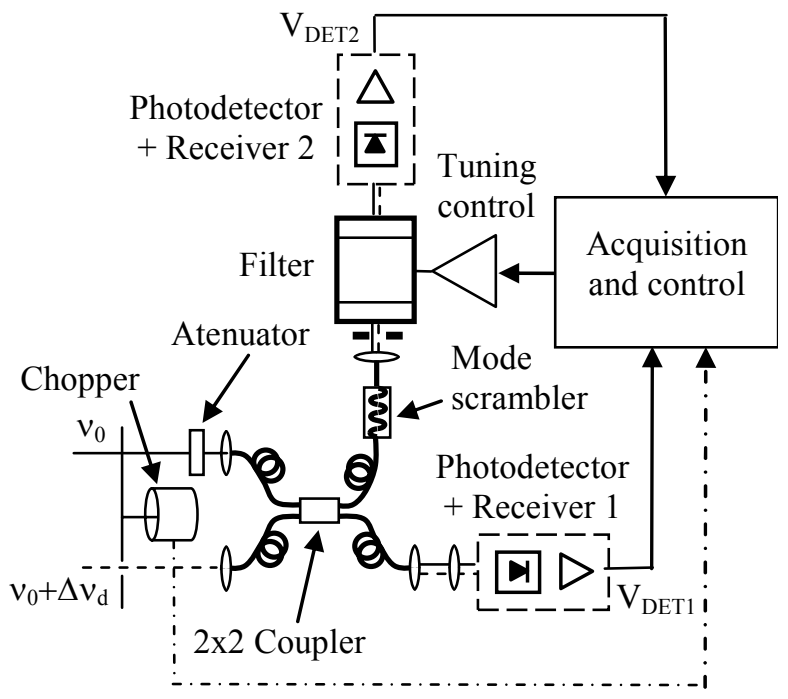

Figure 3. The speed measurement subsystem.

Figure 3 shows the elements of the system used to measure the Doppler shift between emitted and received light. An optical chopper commutates alternatively reference and Doppler channels. Reference channel is attenuated in order to balance power in both optical paths. Focusing lenses are used to couple them into multimode optical fibres. A $2 \times 2$ optical fibre coupler divides the optical power from each channel in two outputs. One of them goes to an avalanche photodiode (APD) based optical receiver through a focusing system formed by two lenses. The other one goes to the high resolution optical filter, in this case a tuneable Fabry-Perot interferometer, and to a second receiver (also APD based). The frequency response of a Fabry-Perot interferometer is extremely sensitive to the incident light characteristics [4]. Hence, a mode scrambler is used to assure the same filter response for both channels (a critical condition to obtain good measurements) by distributing uniformly the optical power among the excited modes in the optical fibre with no dependence on the features of the coupled light in each channel. A collimating lens and a diaphragm condition the incident light to the interferometer. A $16 \mathrm{bits}$, up to $250 \mathrm{ks} / \mathrm{s}$, multifunction acquisition card acquires both receivers output voltage and a personal computer processes data.

As a consequence of the unavoidable drifts in both, the laser wavelength and the resonance frequencies of the interferometer, a real-time control loop has to be set in order to compensate them and maintain the proper relative position between the emitted frequency and the filter slope. This subsystem must act either, on the laser or on the filter. In this case, it will act on the interferometer, which is equipped with piezoelectric cells to fine tune its cavity length and therefore its frequential parameters.

\section{B. Calculations}

If the filter frequency response is precisely characterized during a calibration stage before the measurements, Doppler shift between reference and Doppler channels can be obtained by measuring the corresponding filter transmission, $F\left(v_{0}\right)$ and $F\left(v_{0}+\Delta v_{\mathrm{d}}\right)$. These values can be derived by measuring the output voltage of the receivers, $V_{D E T 1}$ and $V_{D E T 2}$ for each channel.

$$
\begin{gathered}
V_{\text {DET1 }}\left(v_{0}\right)=K_{1} \cdot P_{I N}\left(v_{0}\right) \\
V_{\text {DET2 }}\left(v_{0}\right)=K_{2} \cdot P_{I N}\left(v_{0}\right) \cdot F\left(v_{0}\right) \\
F\left(v_{0}\right)=\frac{K_{1}}{K_{2}} \cdot \frac{V_{D E T 2}\left(v_{0}\right)}{V_{D E T 1}\left(v_{0}\right)} \\
V_{D E T 1}\left(v_{0}+\Delta v_{d}\right)=K_{1} \cdot P_{I N}\left(v_{0}+\Delta v_{d}\right) \\
V_{D E T 2}\left(v_{0}+\Delta v_{d}\right)=K_{2} \cdot P_{I N}\left(v_{0}+\Delta v_{d}\right) \cdot F\left(v_{0}+\Delta v_{d}\right) \\
F\left(v_{0}+\Delta v_{d}\right)=\frac{G_{1}}{G_{2}} \cdot \frac{V_{D E T 2}\left(v_{0}+\Delta v_{d}\right)}{V_{D E T 1}\left(v_{0}+\Delta v_{d}\right)}
\end{gathered}
$$

Where $P_{I N}$ is the incoming optical power and $K_{1}, K_{2}, G_{I}$ and $G_{2}$ are constants including the gain and quantum efficiency of the detectors, the transimpedance gain of the receivers, and losses in the optic elements in each one of the measuring channels. These constants have to be determined in a previous calibration too.

\section{DESIGN CALCULATIONS}

A linear maximum velocity of $33.33 \mathrm{~m} / \mathrm{s}(120 \mathrm{~km} / \mathrm{h})$, an incidence angle to the rotating wheel of $30^{\circ}$ and residual, noncompensated by the tuning control system, short-term frequency drifts of $\pm 1 \mathrm{MHz}$ (measured in the laboratory), have been considered to calculate optimal adjustment of the FabryPerot interferometer in order of assure enough sensitive measurements in all the cases [5]. The result is a full-width 
half-height of the filter of $97 \mathrm{MHz}$ [6]. A proper conditioning of the incoming light allows reaching easily a Finesse of 35 in the Fabry-Perot interferometer. That implies a plate spacing of $44.18 \mathrm{~mm}$ to get the required filter response [4].

\section{RESUlts}

Measurements concerning the tuning control system performance and some speed measurements are going to be presented.

\section{A. Tuning control system performance}

The objective of the tuning control system is to compensate relative frequency drifts between the laser and the filter. Anyway, due to the differential nature of the measurements and as long as measurable frequencies remain in sensitive zones of the slope, certain error in the location of the emitted frequency in the slope of the filter response is going to be possible without important errors in the measurement of frequency drifts. This makes not necessary that the tuning control system acts in a continuous way and for any relative frequency drift.

Two parameters allow controlling these features: 'sensitivity' sets the lower limit of drifts to make the system react and it is defined as a percentage of the change in the filter transmission for the emitted frequency, and 'interval' sets the time between tests and, eventually, tuning correction. Figure 4 shows the behaviour of the system, for different values of 'sensitivity' and 'interval', measured as the standard deviation of the error in the location of the emitted frequency with respect to the optimal value, provoked by small and short-term drifts not compensated by the system. In both cases the time of measurements is 2 minutes.
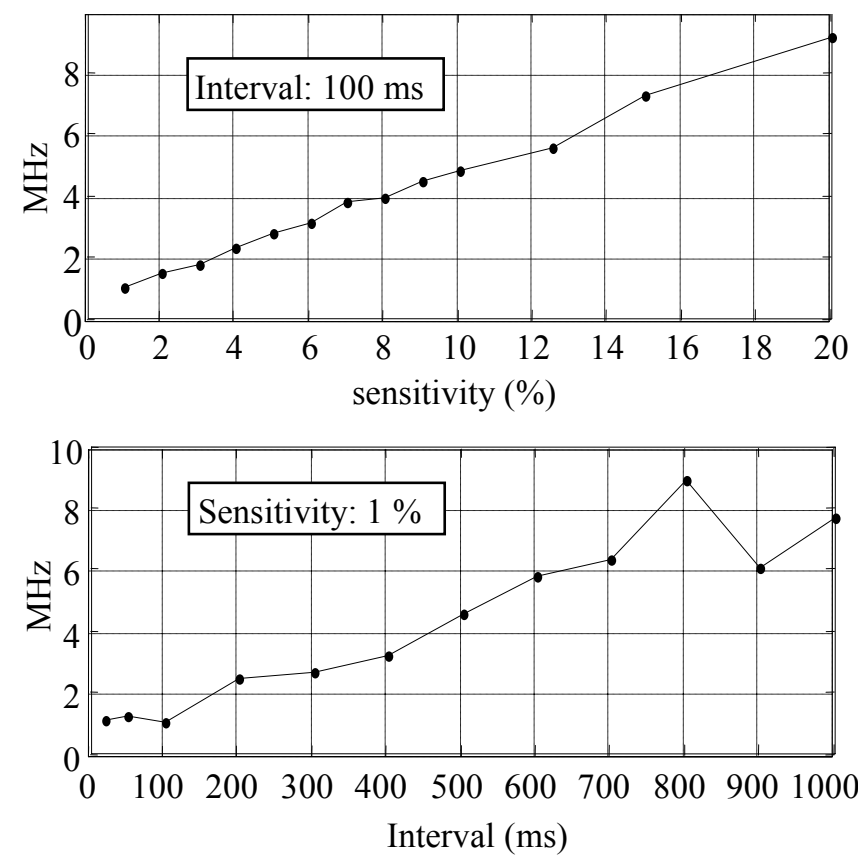

Figure 4. Behaviour of the tuning control system.
As it can be seen, the behaviour is the expected one: the error is growing when either, bigger is its permitted value or longer is the control interval. This measured error has been traduced, by simulations, in loss of sensitivity in speed measurements and has allowed setting a sensitivity of $1 \%$ and an interval of $100 \mathrm{~ms}$ as values that provoke negligible effects.

This configuration has been used to measure the range of short-term non-corrected frequency drifts used in section IV to set the filter configuration.

Monitoring the tuning control voltage applied to the piezoelectric cells has as well allowed calculating long-term relative frequency drifts compensated by the system. The result is $450 \mathrm{MHz}$ amplitude drifts in 15 minutes measurements, that corresponds to more than 4 times the FWHH of the filter. This proves that the tuning control system is indispensable.

\section{B. Speed measurements}

Zero speed measurements using a mirror as target have been made in order to test the accuracy of the measuring system. Figure 5 shows the detected Doppler shift during 2 minutes measurements with 'sensitivity' of $1 \%$ and 'interval' of $100 \mathrm{~ms}$ as tuning control settings. The interferometer has been set up to be able to measure speed up to $\pm 33 \mathrm{~m} / \mathrm{s}$.

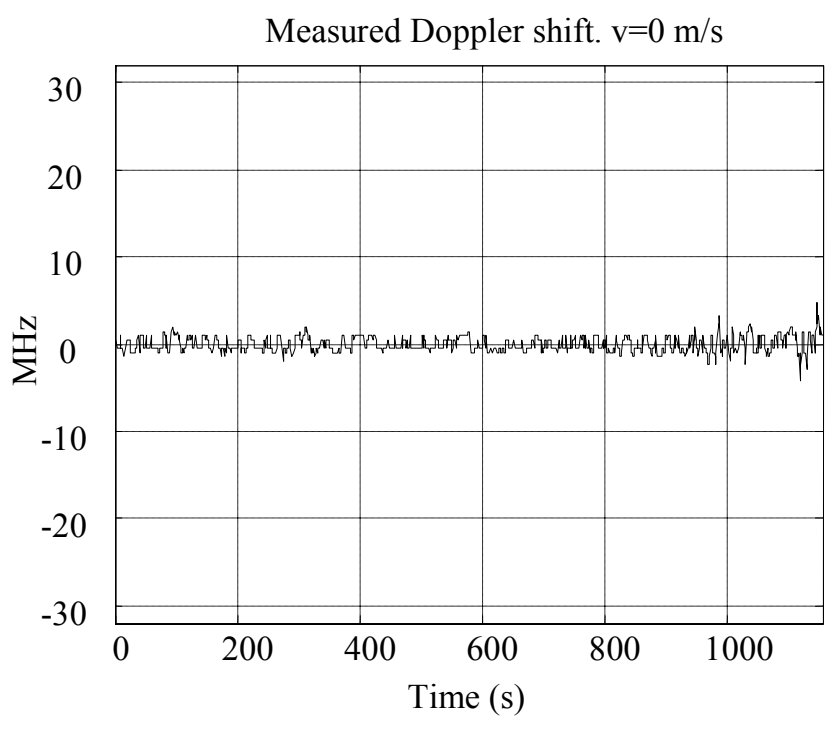

Figure 5. Zero speed measurement.

The standard deviation of the error in the measured Doppler shift is $878 \mathrm{kHz}$ that corresponds to a speed of $0.47 \mathrm{~m} / \mathrm{s}(2.74 \%$ of the maximum measurable speed).

The Fabry-Perot interferometer is not only very sensitive to incident beam features but as well to acoustic noise, mechanical vibrations and thermal effects. Better isolation against these interferences will provide better quality measurements. On another hand, photo-receivers are detecting extremely low levels of optical power and their amplification gain is very high. That makes that electrical interferences are important too and that good protections against them is also a key point in the improvement of the measurements. As it has 
been said in section III, another critical point in this technique, that needs to be optimized too, is the beam conditioning in order to get the same features in both channels. Finally, higher resolution in the filter calibration will also provide better quality measurements.

So far, efficient enough coupling into the optical fiber in the Doppler channel when the rotating wheel is used instead of the mirror, has not been possible and, consequently, non-zero speed measurements are not presented in this paper.

\section{CONCLUSIONS}

A Doppler Lidar continuous-wave velocimeter has been designed and built. A tuning control system has been tested and successfully assessed. It has also been proved that its inclusion in the system is indispensable. Good performance has been obtained in zero-speed measurements. Improvements in order to get non-zero speed measurements and, in general better quality results, are being currently tested.

\section{ACKNOWLEDGMENT}

The authors wish to acknowledge the following entities for partially supporting this research work and lidar systems developed at UPC: European Union and FEDER funds under the EARLINET-ASOS (EU Coordination Action) contract $\mathrm{n}^{\circ}$ 025991 (RICA), and (EU Specific Support Action) contract $\mathrm{n}^{\circ}$ 011863 (RIDS): "Technology development programme towards a European Extremely Large Telescope"; MCYT (Spanish Ministry of Science and Technology) and FEDER funds under the projects TEC2006-07850/TCM and REN200309753-C02-02, Complementary Actions CGL2006-26149E/CLI, CTM2006-27154-E/TECNO, and Special Action REN2002-12784-E; MITYC (Spanish Ministry of Industry, Tourism and Commerce) under the PROFIT project, CIT020400-2005-56. MCYT is also thanked for the Ramón y Cajal position hold by Dr. M. Sicard.

\section{REFERENCES}

[1] C. Laurence Korb, Bruce M. Gentry, and Chi Y. Weng, "Edge technique: theory and application to the lidar measurement of atmospheric wind", Applied Optics. vol. 31, no. 21, pp 4202-4213, 1992.

[2] Matthew J. McGill, James D. Spinhirne, "comparison of two directdetection Doppler lidar techniques", Optical Engineering, 37(10), pp 2675-2686, October 1998

[3] Chanin, M.L., A. Garnier, A. Hauchecorn and J. Porteneuve, “A Doppler Lidar for measuring winds in the middle atmosphere", Geophysical Research Letters, 16, 1273-1276, 1989.

[4] J. M. Vaughan, "The Fabry-Perot Interferometer. History, Theory, Practice and Applications". IOP Publishing, 1989.

[5] Bruce M. Gentry and C. Laurence Korb, "Edge technique for highaccuracy Doppler velocimetry", Applied Optics. vol. 33, no. 34, pp 5770-5777, 1994.

[6] Constantino Muñoz, Alejandro Rodríguez, Adolf Comeron, David Garcia, Francesc Rocadenbosch, Carles Díaz. "Continuous-wave solidtarget doppler lidar prototype". 5th Topical on Optoelectronic Distance/Displacement measurements and Applications. Madrid, October 2-4 2006. Proc. p 204-209. ISBN 84-690-0938-9. 